For Internal Distribution Only

Accelerator Division

Alternating Gradient Synchrotron Department

BROOKHAVEN NATIONAL LABORATORY

Upton, New York 11973

Accelerator Division

Technical Note

AGS/AD/Tech. Note No. 419

NUMERICAL INVESTIGATION OF SPIN

RESONANCE CROSSING WITH SIBERIAN SNAKES

V. Ptitsin*

September 21, 1995

*BINP, Novosibirsk, Russia 


\title{
NUMERICAL INVESTIGATION OF SPIN RESONANCE CROSSING WITH SIBERIAN SNAKES
}

\author{
V.Ptitsin
}

\section{Introduction}

The two pairs of Siberian Snakes will be installed into two rings of RHIC collider, that is under an construction in BNL, to provide a successful acceleration of polarized proton beams to a top energy of $250 \mathrm{Gev}$ [1]. During acceleration, which starts from the injection energy of $25 \mathrm{Gev}$, the beam polarization is affected by numerous spin resonances existing when a frequency of spin precession around the vertical guiding field $\gamma G$ is near $k \pm m \nu_{z}$ where $\nu_{z}$ is a vertical betatron tune and $k, m$ are integers. Siberian Snake, which is generally a special consequence of magnets, rotates a particle spin by $180^{\circ}$ angle around an axis in the horizontal plane. Special arrangements of Snakes in a ring allows to keep the spin tune equal to $1 / 2$ and, therefore, to avoid the spin resonance conditions. However, if the resonance strength is large enough, the spin tune can be shifted noticable from $1 / 2$ value. Moreover, if it is a resonance with the betatron tune (intrinsic) the spin tune becomes modulated with the betatron tune. S. Y. Lee and S. Tepikian found that this modulation effect leads to appearing so-called snake resonances when the fractional part of betatron tune is close to $m / 2 n$ values where $m$ and $n$ are odd integers. They also introduced the good quantative description of spin behavior near the resonance using an envelope function which is in fact a maximum possible deviation for an initially vertically directed spin when observing at a fixed energy (or at a fixed $\gamma G$, what is the same).

Besides the resonance strength, other important factor that determines a result of resonance crossing is the speed a particle goes through the resonance or the acceleration rate. For a common accelerator without snakes the final beam polarization $S_{f}$ after the resonance crossing is described by FroissartStora formula:

$$
S_{f}=S_{i n} \cdot\left(1-\exp \left(-\frac{\pi}{2} \cdot \frac{|w|^{2}}{\alpha}\right)\right)
$$

where $S_{i n}$ is the polarization before a resonance, $|w|$ is the resonance strength and $\alpha=\dot{\gamma} G$ characterizes the energy change rate. So the result of resonance crossing depends strongly on how fast does the energy change comparatively 
with $|w|^{2}$. But, what do we have with snakes? How strong will the beam polarization after the resonance depend on the acceleration rate in this case? In order to answer on this question the numerical investigation of the problem has been carried out and the results of this investigation are presented below.

\section{Tools for tracking}

The SPINK code written by A.Luccio has been used for spin and particle tracking. This code tracks a proton and its spin taking into account horizontal focusing field of quadrupoles, that is in fact the main source of depolarization. The accelerating process is included by an appropriate energy increase after each turn. In the SPINK a snake is a special element which represents a drift interval with a point of view of particle motion and in the same time rotates a proton spin by $180^{\circ}$ around wanted direction in the horizontal plane.

Although the SPINK considers only drifts, bending magnets and quadrupoles, when doing tracking, it still takes the considerable amount of time for the huge RHIC lattice, especially at relatively low acceleration rate $\left(\sim 10^{-5} \mathrm{Gev} / \mathrm{turn}\right)$ when the spin resonance crossing process spreads over some hundreds thousands turns. But as we are investigating the isolated spin resonance, where the spin motion is ruled by one resonance harmonic, it actually does not matter what lattice is analysed. We can investigate more simple lattice instead of the complex one just using an appropriate value of resonance harmonic.

In this work a simple FODO lattice was explored. The lattice consists of 80 FODO cells and the vertical betatron tune was chosen to be in the range between 20.5 and 21 . Thus the sufficient spacing between neighbouring intrinsic resonances was provided and these resonances could be considered as isolated. Two snakes with $\pm 45^{\circ}$ were installed into this lattice. The resonance harmonics were calculated with DEPOL code.

As it follows from [2] a spin behavior near resonance in an accelerator with snakes depends also on the harmonic phase $\phi$ which for an intrinsic resonance can be associated with an initial betatron phase of a particle. Actually one need to know the beam polarization rather than the spin behavior for an individual particle. Hence, the tracking was produced with the use of several particles (generally 10), having the same betatron amplitude but the different betatron phases, and averaged over betatron phase results were taken together with square-root deviations from them.

\section{Results}

\subsection{Far from. snake resonances}

The first round of tracking investigations looked at the situation when the vertical betatron tune was sufficiently far from snake resonance conditions. The 
data were taken at some betatron tune values and at the acceleration rate range from $10^{-5} \mathrm{Gev} /$ turn to $10^{-2} \mathrm{Gev} /$ turn.

A typical spin behavior into the resonance area is shown in Figure 1 for $|w|=0.24$ and $\dot{E}=5 \cdot 10^{-5}$. Although the spin deviates considerably from vertical direction when proton energy approaches the resonance value, far right from resonance it restores again. It is an good example of clearly adiabatic behavior. Without snakes at the same values of $|w|$ and $\dot{E}$ according to FS formula we have also adiabatic crossing of the resonance, with spin flip in that case. Actually, the presence of snakes do not only preserve the adiabaticity but considerably enhance it. It is demonstrated in Figure 2 where very high acceleration rate $\dot{E}=5 \cdot 10^{-2}$ was taken. The FS formula gives in this case the large polarization loss. But with snakes there is no any polarization loss and the spin behavior is practically the same as at $\dot{E}=5 \cdot 10^{-5}$. The collected data showed no the loss of polarization at any reasonable values of the parameters. The depolarization begin only at unreally high acceleration rate $\left(\dot{E}>10^{-1}\right)$ or at sufficiently large resonance strength $(|w| \geq 1)$ (Fig.3). But according to the DEPOL calculation the maximum resonance harmonic on RHIC would be less than 0.5 .

It should be noted also that due to the adiabatic character of spin resonance crossing the description involving an static envelope function [2] to figure out the spin deviation into the resonance zone works well.

If we will lower the value of resonance harmonic $|w|$ a spin deviation from vertical direction will decrease rapidly. Figure 4 demonstrates the dependence of the maximum spin deviation on the resonance strength. The resonances with harmonics less than $5 \cdot 10^{-2}$ appear to be unimportant. It is what the application of snakes actually does: removing the small resonances and adiabatic crossing strong ones.

As it has been noted above the adiabaticity is broken at sufficiently large resonance strength. Another case when the beam polarization can be lost is snake resonances near specific values of vertical betatron tune. I have investigated the latter case because of its more importancy for RHIC.

\subsection{Betatron tune near the snake resonance condition}

For the investigation the snake resonance when fractional part of betatron tune approach to $5 / 6$ was chosen. At RHIC the vertical betatron tune is planned to be about 28.83 that is close to this resonance value.

Near the snake resonance there is one more parameter, namely the detuning $\delta$ from the exact snake resonance value, which affects the result of resonance crossing. Thus the dependence of final beam polarization on $|w|, \dot{E}$ and $\delta$ has been studied. For example, Figure 5 demonstrates a clear dependence of the polarization after resonance crossing on the acceleration rate. The collected data shows a very different character of this dependence in various regions of the parameter space. At sufficiently large detuning $\delta$ the dependence has a 
very regular form (Fig.6) with a minimum value of the final polarization at some acceleration rate. But when the betatron tune goes closer to the snake resonance condition the regular picture is broken and the final polarization depends on the acceleration rate in very complex, chaotic-like way (Fig.7). The same non-regular region one can find by scanning the resonance harmonic value (Fig.8).

Nevertheless at any values $|w|$ and $\delta$ one can find such a value of acceleration rate below that the polarization after crossing of the resonance is preserved. Another words there is a region in three dimensional parameter space $(|w|, \alpha, \delta)$ where the adiabaticity condition continue to be satisfied. I numerically found the border of this region corresponding to the $1 \%$ loss of polarization. It can be presented as in Figure 9 where in the $\delta-\alpha$ plane the curves are drawn corresponding to the border at various values of resonance harmonics. The right-down side from the curves is the good region. For RHIC collider, with $4 \cdot 10^{-5} \mathrm{Gev} /$ turn acceleration rate, we conclude that the detuning from the snake resonance value must be larger than $10^{-3}$. Although, in principle, the vertical betatron tune can approach closer to the snake resonance value if we decrease the acceleration rate.

\section{Conclusion}

In this report the results of numerical simulations of spin resonance crossing with Siberian Snakes are presented. It is shown that, when the vertical betatron tune is far from the snake resonance values, the spin resonance is crossed adiabatically for the expected resonance harmonic range on RHIC $(|w|<0.5)$ and no polarization loss happens. Near the snake resonance conditions there is the complex dependence of the final polarization on the harmonic value, the acceleration rate and detuning from the snake resonance value. The border of the region has been found where the beam polarization is preserved.

\section{Acknowledgements}

I would like to thank E.Courant, A.Luccio and T.Roser for many helpful discussions in the process of this work.

\section{References}

[1] Conceptual Design for the Acceleration of Polarized Protons in RHIC

[2] S. Y. Lee and S. Tepikian Phys. Rev. Lett., 56, 1635 (1986) 


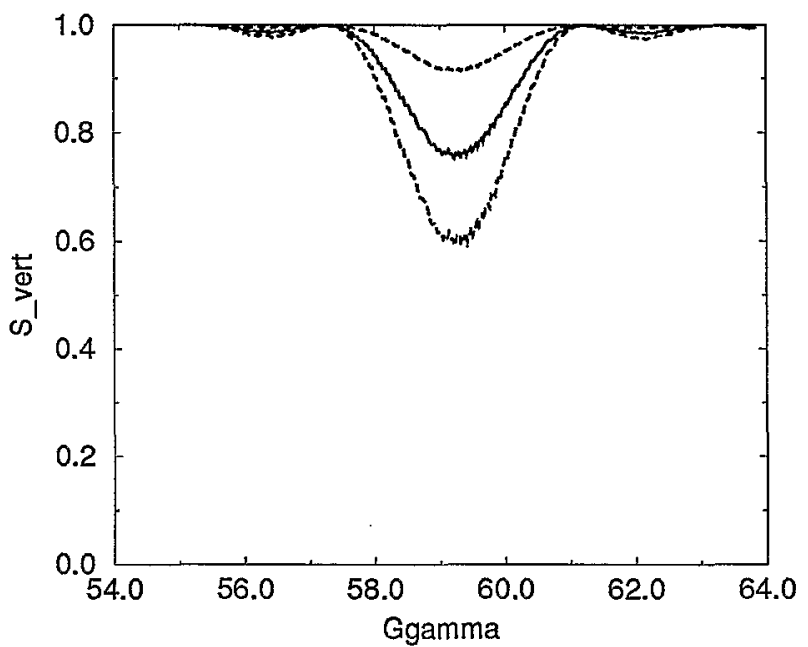

Figure 1: Crossing the resonance $\gamma G=59.24,|w|=0.24, \dot{E}=5 \cdot 10^{-5}$. Solid line - the polarization averaged over betatron phase, dashed - square root deviations from average.

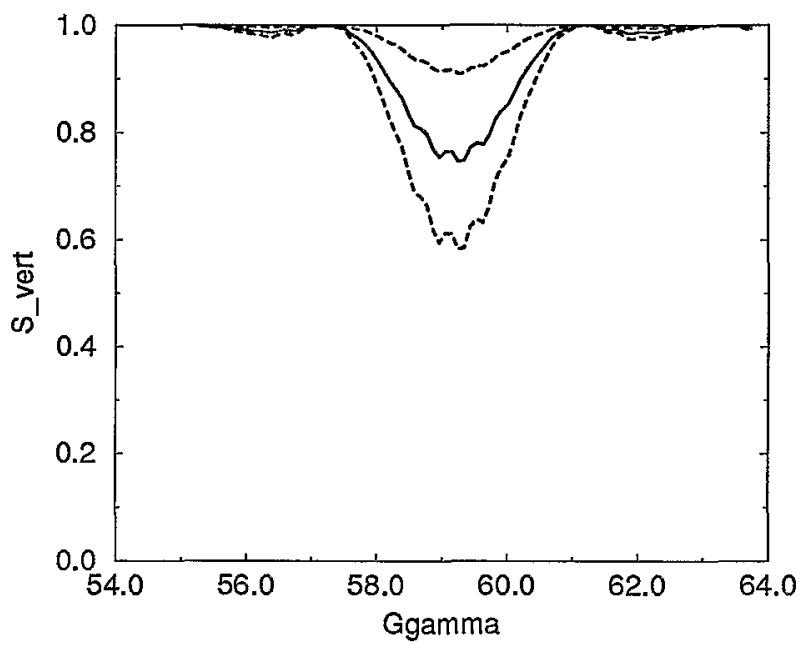

Figure 2: Crossing the resonance $\gamma G=59.24,|w|=0.24, \dot{E}=5 \cdot 10^{-2}$. Solid line - the polarization averaged over betatron phase, dashed - square root deviations from average. 


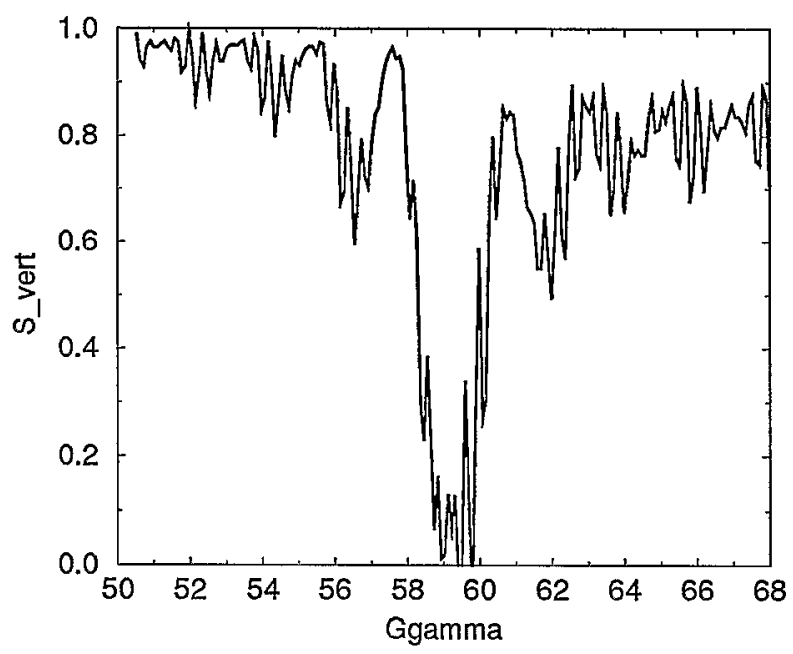

Figure 3: Crossing the resonance $\gamma G=59.24,|w|=1.2, \dot{E}=5 \cdot 10^{-2}$.

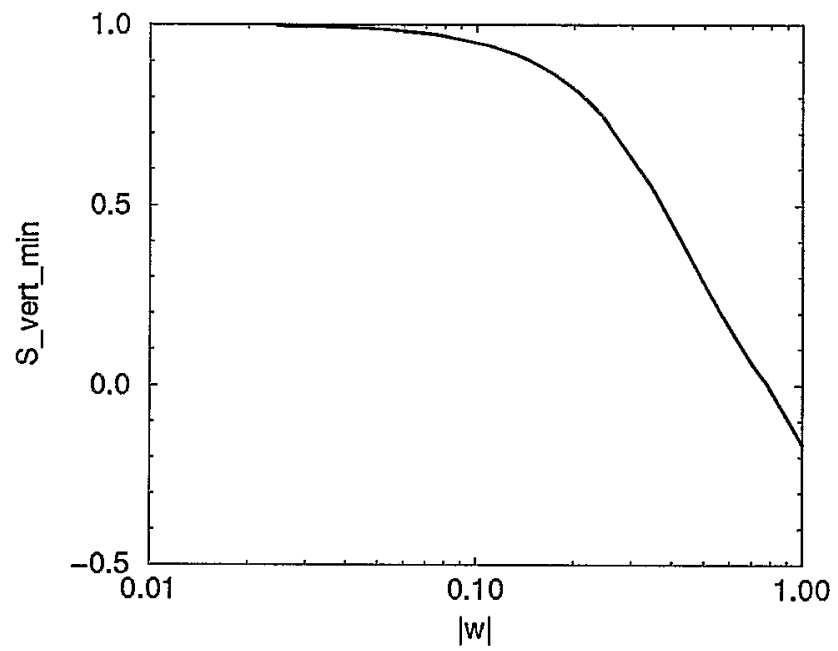

Figure 4: Maximum spin deviation into the resonance region versus the resonance harmonic value. 


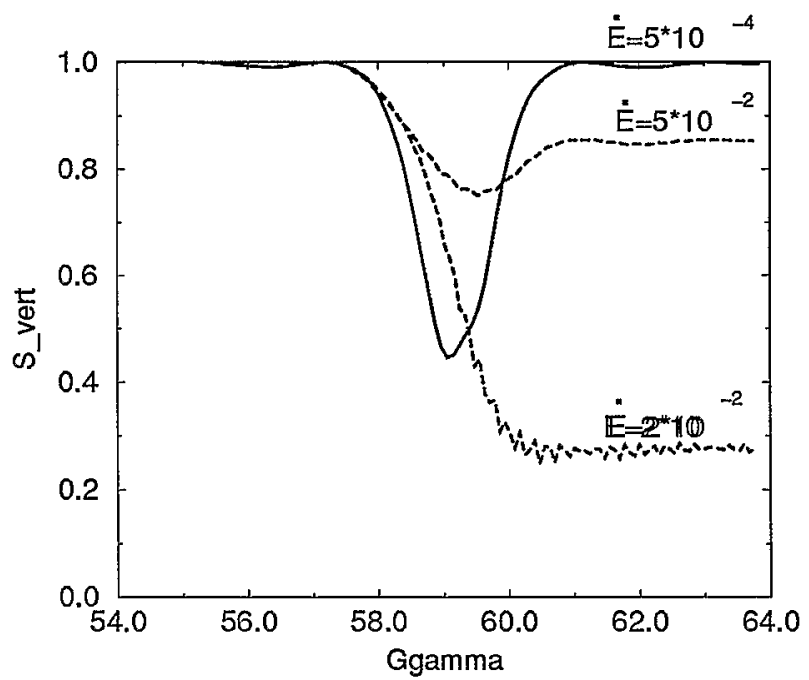

Figure 5: Various results of resonance crossing for various acceleration rates. $\delta=2.5 \cdot 10^{-3},|w|=0.245$

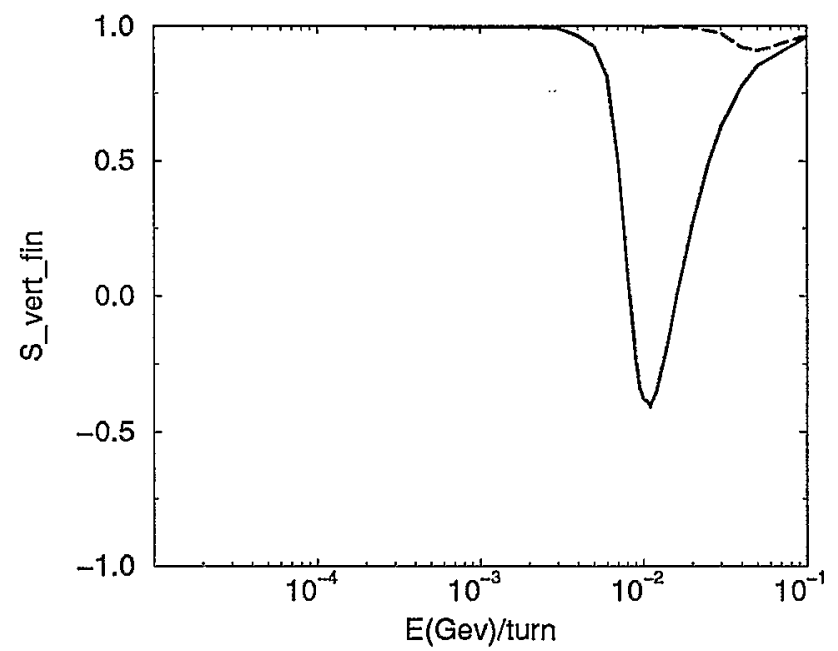

Figure 6: The dependence of final polarization on the acceleration rate for $\delta=2.5 \cdot 10^{-3}$ (solid) and $\delta=1.2 \cdot 10^{-2}$ (dashes). $|w|=0.245$. 


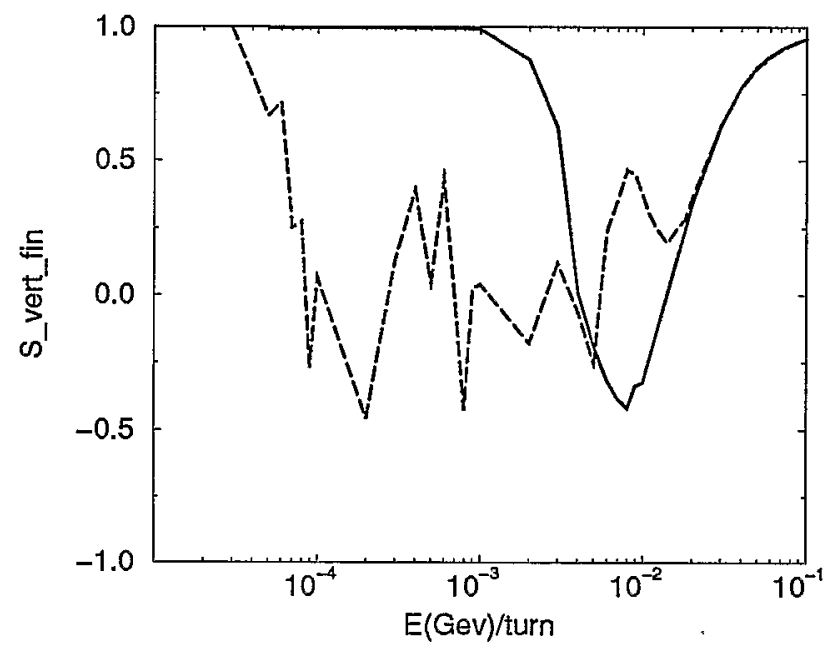

Figure 7: The dependence of final polarization on the acceleration rate for $\delta=1.2 \cdot 10^{-3}$ (solid) and $\delta=2.5 \cdot 10^{-4}$ (dashes). $|w|=0.245$.

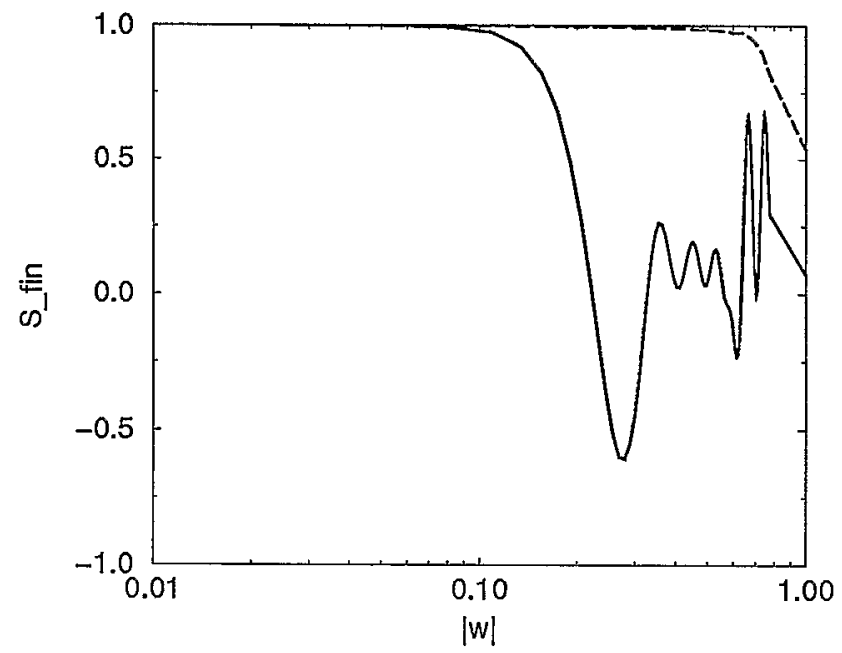

Figure 8: The dependence of final polarization on the value of resonance harmonic at $\dot{E}=1 \cdot 10^{-2}$ (solid line) and $\dot{E}=5 \cdot 10^{-4}$ (dashed line) for $\delta=2.5 \cdot 10^{-3}$. 


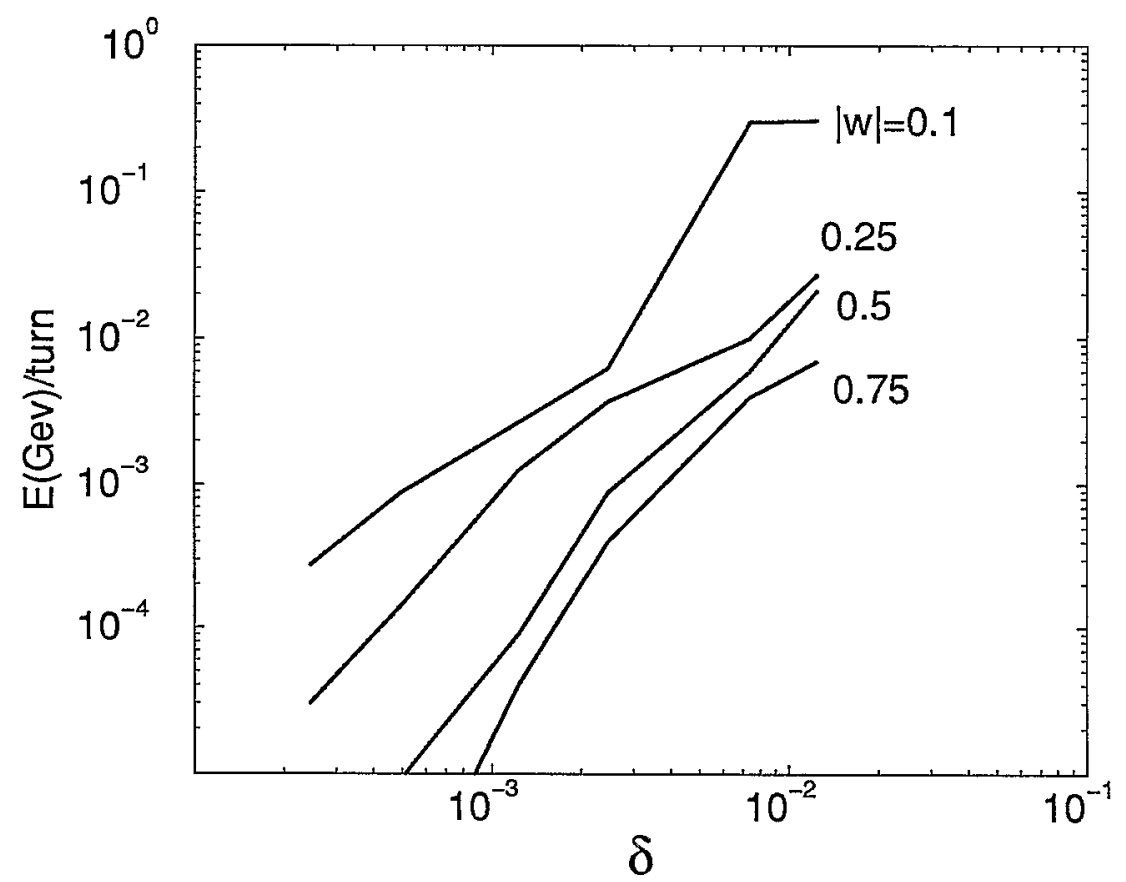

Figure 9: The border of a region with less than $1 \%$ polarization loss for various resonance strength. 